\title{
DICOM Medical Image Browser Design and Implementation Based on iOS System
}

\author{
Bao Nan \\ School of Sino-Dutch Biomedical \& Information \\ Engineering \\ NEU \\ Shenyang, China \\ baonan@bmie.neu.edu.cn \\ Liu Cheng \\ School of Sino-Dutch Biomedical \& Information \\ Engineering \\ NEU \\ Shenyang, China \\ 531788374@qq.com
}

\author{
Li Yao \\ School of Sino-Dutch Biomedical \& Information \\ Engineering \\ NEU \\ Shenyang, China \\ 501736413@qq.com \\ Zhong Xu Xiang \\ School of Sino-Dutch Biomedical \& Information \\ Engineering \\ NEU \\ Shenyang, China \\ 1016985603@qq.com
}

\author{
Kang Yan* \\ School of Sino-Dutch Biomedical \& Information Engineering \\ NEU \\ Shenyang, China \\ kangyan@bmie.neu.edu.cn
}

\begin{abstract}
Now, the iPhone/iPad have been widely used in the daily life. If we can apply this into the healthcare field, it will improve the efficiency for the doctor to scan the medical images information. In this case, we designed and developed a DICOM image portable browsing terminal system based on the iOS platform. It was developed by the Xcode, and contains the features that data synchronization, DICOM files parsing, multi-planar reconstruction(MPR), transferring the window, zoom, and translation of DICOM images. Because the portable devices possess several limitations relatively to PCs, such as low frequency, low memory and poor computing, etc, we used a multiplanar reconstruction accelerated method based on the incremental coordinate' transformation calculation method, which has greatly improved the multi-planer reconstruction speed, and the fluency and operability of the system,. All of them have made the system extremely conveniently, stability and maneuverability. It can be widely used in the whole machine of iOS system for the doctor intelligent diagnostic, has great clinical application value.
\end{abstract}

Keywords: iOS; The image browser; MPR; Increment coordinate; DICOM analysis

\section{INTRODUCTION}

With the development of medical imaging technology, many hospitals in our country are acquiring a variety of digital imaging equipment such as CT, MRI, ultrasound, PET and so on. These devices have an interface of output images in DICOM format. However, the large-scale image processing workstations or treatment planning systems of the domestic hospitals require a large consumption of the hardware configuration, thus limiting the use of medical images [1]. Besides, most of the supporting medical imaging workstations are based on the Windows operating system [2], so that doctors are confined to wok by their desktops, greatly decreasing the flexibility.

With the development and popularization of smart phones and tablet PCs whose corresponding software market is also expanding, medical image processing running on smart devices becomes possible. Currently, some well-known software of medical image processing based on iOS appears in iTunes, such as 3D4Medical Image, iClarity Lite, ImageVis3D Mobile Universal and so on. By now, FDA (U.S. Food and Drug Administration) has authorized iPad / iPhone to be ancillary products of medical imaging and radiology diagnostic. But the quantity of domestic application software of medical imaging based on iOS is much smaller, and the relevant techniques are relatively poor [2].

The purpose of this paper is to combine demands and technologies. In order to extend the range of applications of portable devices and increasing the flexibility of medical imaging diagnostic A DICOM medical imaging viewer based on iOS had developed, which achieves the DICOM data synchronization, DICOM images and 
information display, images multi-manipulation, multiplanar reconstruction and other functions. The viewer released on iTunes is named MedInsight. The application provides convenience for doctors browsing images of the patients while reduces the dependence on large scale workstations, and implements the portable reading images and diagnostics, which not only reducing the cost, but also enhancing the flexibility and practicality.

\section{SYSTEM DESIGN AND ANALYSIS}

Basic functions of MedInsight include DICOM data synchronization, DICOM files analysis, basic manipulations on medical images, multi-planar reconstruction etc. Its functional framework is shown in Fig. 1:

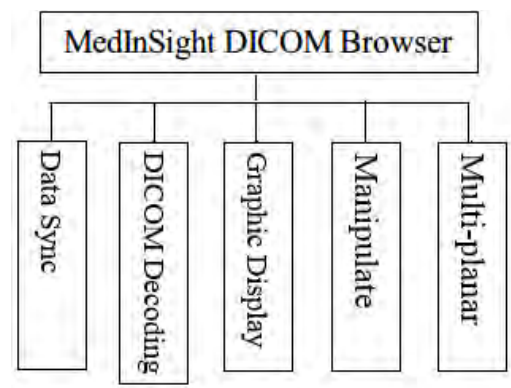

Figure 1. Browser functionality framework

DICOM files are synced to iPad or iPhone via iTunes, and read by the application when it launch. Through multi-planar reconstruction, the application can present horizontal, vertical, coronal and any angle of surfaces of the image. Meanwhile pan, zoom and adjust the window center and width can be done through assistive Touch, to obtain the corresponding information of the image.

\section{SYSTEM IMPLEMENTATION}

\section{A. DICOM Files Analysis and Display}

With the widespread use of digital medical, in particular the development of PACS [3] (picture archiving and communication system) and telemedicine systems, the standard of DICOM comes into being. DICOM is the abbreviation of Digital Imaging and Communication of Medicine, which is a standard name for the storage and transmission of medical images designated by American College of Radiology and the American Electrical Manufacturers Association. Currently DICOM 3.0 version it's mainly used. The standard has now been widely applied to medical profession, which has become an international common standard in the field of medical imaging information [4].

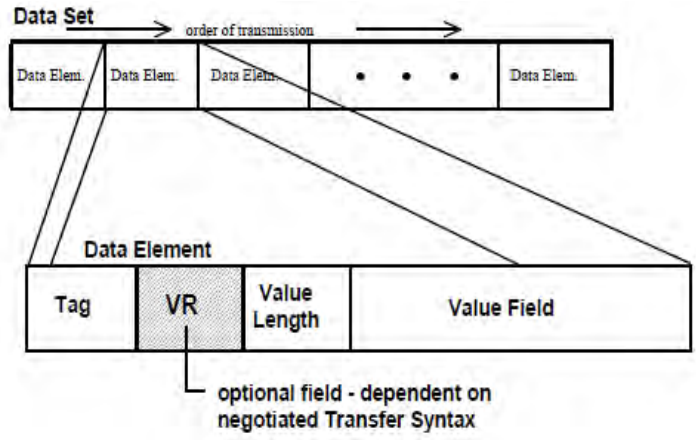

Figure 2. DICOM file structure

As Fig. 2 shows, a DICOM file commonly consists of a DICOM header and a DICOM data set. Its format information is shown as follows:

1) $\mathrm{DICOM}$ Header

A DICOM header contains the file sequence, transfer syntax, the prefix and the patient information.

\section{2) DICOM Data Set}

It's formed by the DICOM data elements (Data Element) arrayed in a certain order, which can be divided into image file data and non-image file data according to the content.

Among four fields of data elements, Tag is the only code consists of a 16-bit group number and a 16-bit unit number. Main data elements from format image file of DICOM 3.0 is shown in Table 1:

TABLE I. DICOM IMAGE IMPORTANT TAG MARKING INFORMATION

\begin{tabular}{|l|c|}
\hline \multicolumn{1}{|c|}{ Tag Name } & Tag \\
\hline Transfer Syntax UID & $(0002,0010)$ \\
\hline Rows & $(0028,0010)$ \\
\hline Columns & $(0020,0011)$ \\
\hline Bits Allocated & $(0028,0100)$ \\
\hline Window Center & $(0028,1050)$ \\
\hline Window Width & $(0028,1051)$ \\
\hline
\end{tabular}

Using Xcode development environment and programming with Objective-C, the application read and analyze file characters in DICOM files to obtain sequence information and image data, which are used to construct a three-dimensional data volume. Then corresponding BMP images are generated using adjusted window center and width and are displayed on the iOS system.

\section{B. Multi-planar Reconstruction}

By scanning with CT, MRI or PET, we can get an image sequence. Except for the positioned image, images parallel to each other, and there is a certain distance between the layers. Normally, the program reads sequences layer by layer and stores data in the three-dimensional data volume. MPR (Multi-planar Reconstruction) [6-7] reconstructs areas designated by certain segments and curves from coronal, vertical, and horizontal position and any angle section of the image in the three-dimensional data volume, extracting the appropriate two-dimensional image data from the threedimensional image data. In the process of rebuilding, there is missing data which is necessary to take the methods of interpolation. The most commonly used 
methods of interpolation are neighborhood interpolation, linear interpolation, spline interpolation, etc. [8-9]. Taking into account the effects and optimization of resource consumption, the most common method, neighborhood interpolation, is used here. In order to obtain data from oblique sections according to the azimuth information, the principles of MPR reconstruction are explained as follows.

1) Multi-planar Reconstruction Method Based on Traditional Coordinate Transformation

Commonly the traditional algorithm of MPR reconstruction is to transform the original image into reference image in the three-dimensional data body by using the coordinates rotation and translation. Using the homogeneous matrix to represent a point $(x, y, z)$ in

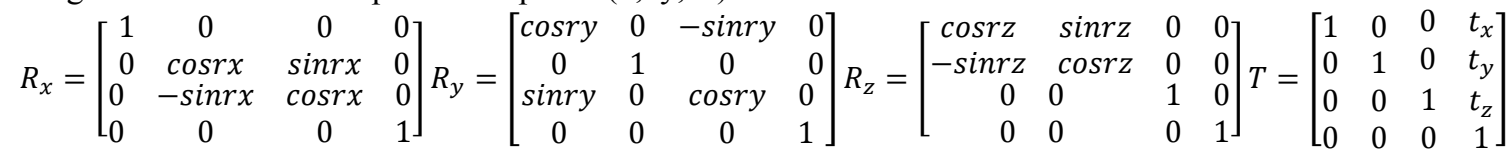

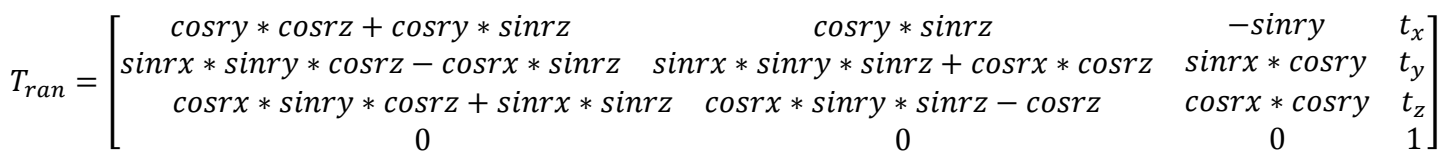

Since the transformation is from the two-dimension to the three-dimension, so that there isn't the z-axis coordinate data in original data. Converted into the three-dimensional space, at least 20 multiplications and components of coordinates for once. Each point in a two-dimensional image has to do the corresponding rotation transformation. Hence, if to generate a $512 *$ 512 image, the number of additions and multiplications is about $512 * 512 *(20+13)$ in total, which requires a large amount of calculation.

2) Multi-planar reconstruction Method Based on Rapid Transformation Coordinates' Increment

Since both of distance and angle between each point of the image section keep constant, irrelevant to the rigid transformation, the grid unit length shows a linear relationship before and after the transformation. According to this feature, we can traverse the image by using the increment of coordinates to simplify the transformation of each point and thus to speed up the overall coordinates transformations. Therefore, we use the method based on the coordinates' increment to accelerate computing coordinates' transformation [10$11]$.

As shown in Fig. 3, in the two-dimensional coordinate system, first determine the rotation center of the image P00 (x00, y00), and calculate the reference point in the new coordinate $\mathrm{P} 00(\mathrm{x} 00 *$, y00*). Then calculate the coordinates' increment corresponding to the $\mathrm{x}, \mathrm{y}$ axis in the new coordinate system of the grid length $\mathrm{dx}$, dy along the $\mathrm{x}, \mathrm{y}$ axis in the original image. Finally, the new coordinates of all other points can be directly determined by changing the coordinates' increment . 13 additions are required when transform the transformation:

$$
P_{a}=T_{\text {ran }} * P_{b}
$$

The coordinates vector of the origin image in the formula is $\mathrm{Pb}=[\mathrm{x}, \mathrm{y}, \mathrm{z}, 1]^{\mathrm{T}}$. The coordinates vector of the reference image is $\mathrm{Pa}=\left[\mathrm{x}^{*}, \mathrm{y}^{*}, \mathrm{z}^{*}, 1\right]^{\mathrm{T}}$. The transformation matrix is $\mathrm{T}_{\text {ran }}=\mathrm{T} \cdot \mathrm{R}$ wherein $\mathrm{R}$ can be decomposed into $\mathrm{Rx}, \mathrm{Ry}, \mathrm{Rz}$, the $4 \mathrm{x} 4$ transformation matrix respectively rotating around the $\mathrm{x}, \mathrm{y}, \mathrm{z}$-axis. If the rotation angles respectively are rx, ry and $\mathrm{rz}$ in radians, and the order of rotation is firstly around the $\mathrm{Z}$ axis, then around the $\mathrm{Y}$ axis, and finally around the $\mathrm{X}$ axis, thus, $\mathrm{R}=\mathrm{Rx} \cdot \mathrm{Ry} \bullet$ $\mathrm{Rz} . \mathrm{T}$ is a translation and rotation matrix whose equation can be expressed as: space, to get the new coordinate $\left(x^{*}, y^{*}, z^{*}\right)$ after
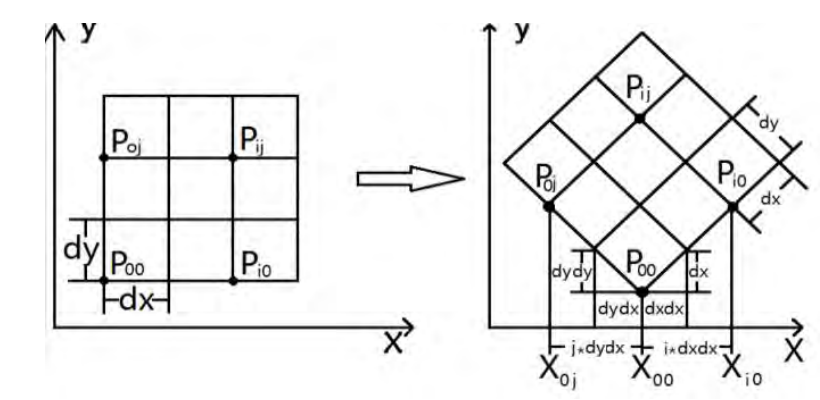

Figure 3. Incremental coordinate transformation method

As Fig. 3 shows, in the new coordinate system, the coordinate of the arbitrary point $\mathrm{PiO}$ in the first line is:

$$
\left\{\begin{array}{l}
x_{i 0}^{*}=x_{00}^{*}+d x d x \cdot \mathrm{i} \\
y_{i 0}^{*}=y_{00}^{*}+d x d y \cdot \mathrm{i}
\end{array}\right.
$$

In the formula, $\mathrm{i}$ represents the $\mathrm{x}$-axis coordinates and $\mathrm{j}$ is the $\mathrm{y}$-axis coordinates in the reference image corresponding to the original image. $\mathrm{xi}^{*}$ and $\mathrm{yi} 0^{*}$ are the new coordinates in the reference image. $\mathrm{dxdx}$ and dydy are respectively the increments of the grid unit length along the $\mathrm{x}$ and $\mathrm{y}$ axis in the reference image transformed corresponding to the grid unit length $\mathrm{dx}$ along the $\mathrm{x}$ axis in the original image.

Similarly, any point Pij(xij, yij) can be transformed to become the new one Pij(xij*, yij*) in the reference image according to:

$$
\left\{\begin{array}{l}
x_{i \mathrm{j}}^{*}=x_{\mathrm{i} 0}^{*}+d \mathrm{y} d x \cdot \mathrm{j} \\
y_{i \mathrm{j}}^{*}=y_{\mathrm{i} 0}^{*}+d \mathrm{y} d y \cdot \mathrm{j}
\end{array}\right.
$$

Thus, the three-dimensional coordinate rotation formula can be derived via the same thoughts, as shown in Fig. 4. 


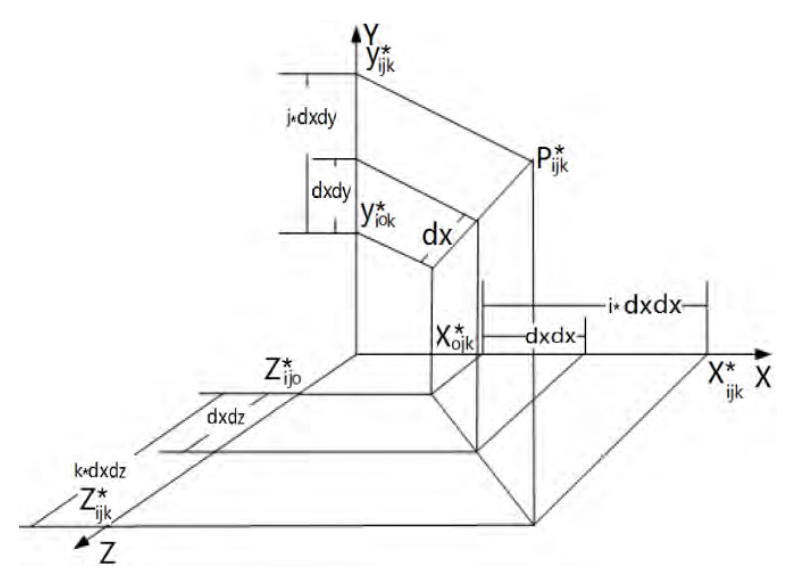

Figure 4. Three dimensional incremental coordinate transformation method

The formula of calculating the new coordinates of any point Pijk from any position $\mathrm{i}, \mathrm{j}, \mathrm{k}$ of the original coordinates is:

$$
\left\{\begin{array}{l}
x_{i j k}^{*}=x_{0 j k}^{*}+d x d x \cdot i \\
y_{i j k}^{*}=y_{0 j k}^{*}+d x d y \cdot i \\
z_{i j k}^{*}=z_{0 j k}^{*}+d x d z \cdot i
\end{array}\right.
$$

Wherein, (x0jk *, y0jk *, $\mathrm{z} 0 \mathrm{jk} *$ ) is the first coordinate of the the $\mathrm{j}$-th row of the k-th plane in the original coordinate system.

$$
\left\{\begin{array}{l}
x_{0 j k}^{*}=x_{00 k}^{*}+d y d x \cdot j \\
y_{0 j k}^{*}=y_{00 k}^{*}+d y d y \cdot j \\
z_{0 j k}^{*}=z_{00 k}^{*}+d y d z \cdot j
\end{array}\right.
$$

Wherein, $\left(\mathrm{x} 00 \mathrm{k}^{*}, \mathrm{y} 00 \mathrm{k}^{*}, \mathrm{z} 00 \mathrm{k}^{*}\right)$ is the new coordinate corresponding to the first point in the $\mathrm{k}$-th plane of the original coordinate system, which can be calculated by:

$$
\left\{\begin{array}{l}
x_{00 k}^{*}=x_{000}^{*}+d z d x \cdot k \\
y_{00 k}^{*}=y_{000}^{*}+d z d y \cdot k \\
z_{00 k}^{*}=z_{000}^{*}+d z d z \cdot k
\end{array}\right.
$$

From formula 2.3 , the formula of coordinates' increment transformation in the three-dimension is:

$$
\begin{aligned}
& \left\{\begin{array}{l}
d x d x=d x \cdot \cos r y \cdot \cos r z \\
d x d y=d x \cdot \cos r y \cdot \sin r z \\
d x d z=d x \cdot(-\sin r y)
\end{array}\right. \\
& \left\{\begin{array}{l}
d y d x=d y \cdot(\sin r x \cdot \sin r y \cdot \cos r z-\cos r x \cdot \sin r z) \\
d y d y=d y \cdot(\sin r x \cdot \sin r y \cdot \sin r z+\cos r x \cdot \cos r z) \\
d y d z=d y \cdot \sin r x \cdot \cos r y
\end{array}\right. \\
& \int d z d x=d z \cdot(\cos r x \cdot \sin r y \cdot \cos r z+\sin r x \cdot \sin r z) \\
& \{d z d y=d z \cdot(\cos r x \cdot \sin r y \cdot \sin r z-\sin r x \cdot \cos r z) \\
& d z d z=d z \cdot \cos r x \cdot \cos r y
\end{aligned}
$$

$\mathrm{dx}$, dy and $\mathrm{dz}$ represent the grid unit lengths in the original image. Because the data from the z-axis don't have to be taken into consideration when rebuilding into the two-dimension, thus $\mathrm{dz}=0$. The final result can be combined as:

$$
\left\{\begin{array}{l}
x_{i j k}^{*}=x_{000}^{*}+d x d x \cdot i+d y d x \cdot j \\
y_{i j k}^{*}=y_{000}^{*}+d x d y \cdot i+d y d y \cdot \mathrm{j} \\
z_{i j k}^{*}=z_{000}^{*}+d x d z \cdot i+d y d z \cdot \mathrm{j}
\end{array}\right.
$$

Before the transformation, the coordinates' increment can be calculated merely when knowing the values, $\mathrm{dxdx}, \mathrm{dxdy}, \mathrm{dydz}$, dydy and dydz. So by using the new method, calculating each point only requires 6 additions and 6 multiplications. If to generate a $512 * 512$ image, there will be a calculation of $512 * 512 *(6+6)$ times, which greatly increase the speed of the reconstruction.

\section{THE RESULTS OF EXPERIMENTS}

\section{A. Overall Results}

Define abbreviations and acronyms the first time they are used in the text, even after they have been defined in the abstract. Abbreviations such as IEEE, SI, MKS, CGS, sc, dc, and rms do not have to be defined. Do not use abbreviations in the title or heads unless they are unavoidable.

The first generation of iPad(16G WiFi) whose operating system is iOS5.1(9B176), duel-core processor, RAM 512MB is used in this experiment with Xcode4.3.2 development environment and Objective-C. MedInsight DICOM viewer finally comes into being. The experimental data is DICOM sequences from 250 images, $512 * 512 * 250$. After importing DICOM medical image data through the device port, the program generates the three-dimensional data body. The sequence images can be displayed through multiple windows after the process calculation, as Fig. 5 shows.

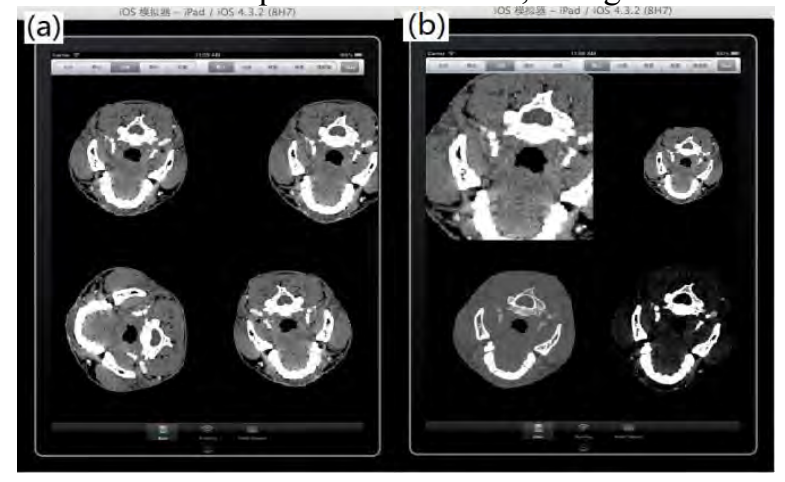

Figure 5. Multi-function image manipulation

At the same time, besides the corresponding multiple operations, horizontal, coronal and vertical images can be calculated and displayed by reading the threedimensional data body, as Fig. 6 shows. In addition, to display arbitrary angle of sections of the image can be displayed via multi-planar reconstruction algorithm, as Fig. 7 shows. 


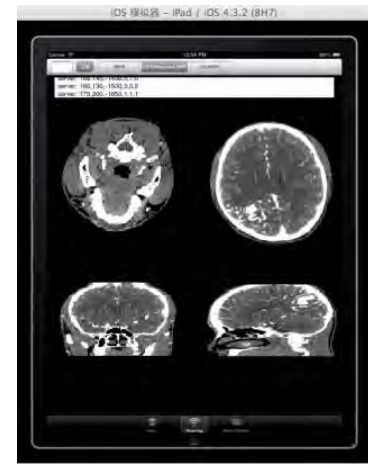

Figure 6. Three axis images

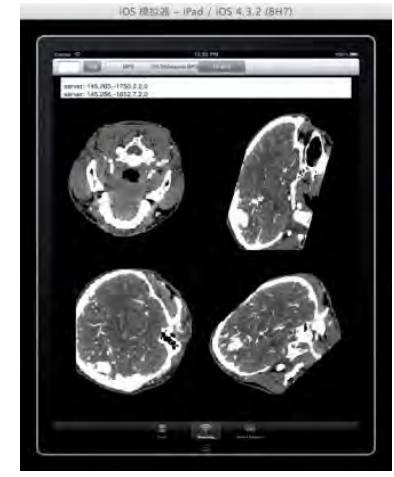

Figure 7. MPR model images

\section{B. The Comparison between the Speed of Traditional} and Improved Algorithm of MRI

In order to compare the effects and time of generating MRI images between two algorithms, we do the experiments as follows: The devices mentioned in 3.1 are used. Then set the central point $(256,256,125)$ as the starting point in the three-dimension. So, we get the level relations as Fig. 8 shows by rotating the horizontal plane which is through the starting point as the starting plane.

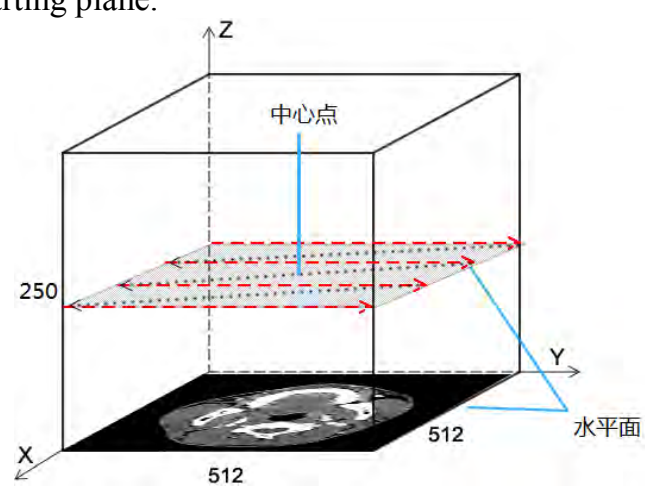

Figure 8. Horizontal plane orientation figure

Rotation interval is selected as $5^{\circ}$, regardless of the symmetry, we need to rotate to $180^{\circ}$, and the results are $36 * 36$ image data and the time data. Time data's unit is milliseconds (ms).

To guarantee the rigorousness of the comparison, the similarity must be compared first between two algorithms. From formula (3) and (12), two algorithms are the same theoretically. But due to the numerical calculation and precision limit, there are usually differences from the images generated by two algorithms. So, calculate the different number of pixels $\mathrm{D}$ of the images, as Fig. 9 shows the different number of points in a horizontal plane.

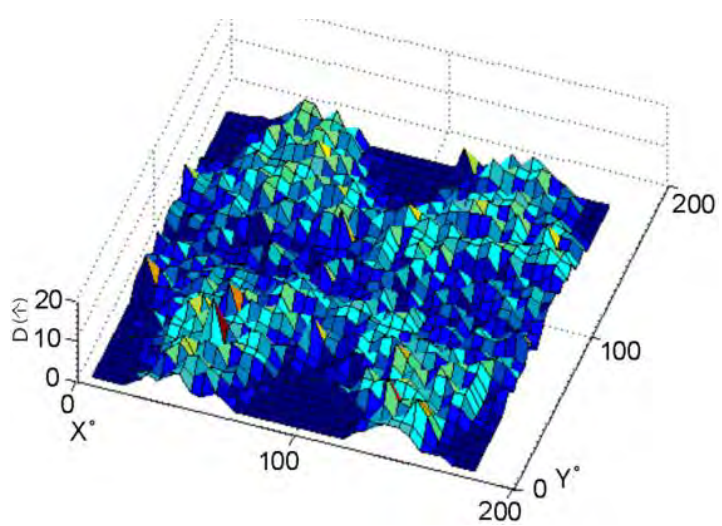

Figure 9. The difference number distribution of two algorithms

As Fig. 9 shows, when the section parallels with three-axis planes, images are basically the same generated by two algorithms. With the increment of the angle and section's area, the number of distinct points will increase.

Take account of three different kinds of planes, and collect data for 50 times repeatedly. After averaging, the numbers of points which are different from each other are $10.23,9.45$ and 9.38 (the average is 9.69), when horizontal, vertical and coronal planes are regarded as the starting plane respectively. The result illustrates that if generate a $512 * 512$ image, the proportion of the distinct points is $0.37 \%$, which means images generated by two different algorithms can be considered as the same.

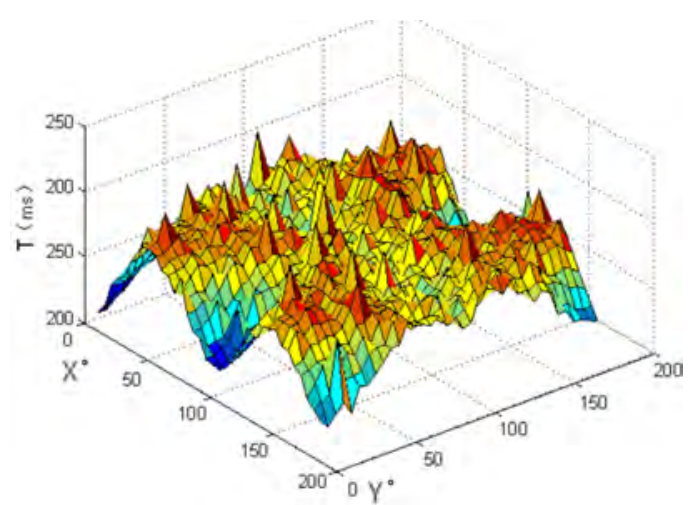

Figure 10. The time distribution of traditional algorithm

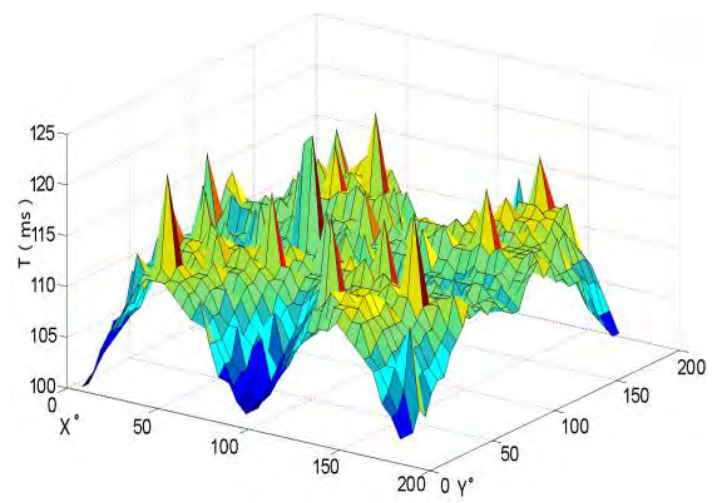

Figure 11. The time distribution of improved algorithm 
As Fig. 10 and Fig. 11 show, when the section parallels with three-axis planes, it take the shortest time. With the increment of the angle and section area, the calculate time will increase. Which corresponds to the reality. Combine different cases of 3 different planes, repeat collecting data for 50 times and do the averaging. The results are shown in the following table.

TABLE II. RESULTS OF TWO ALGORITHMS`CALCULATION TIME

\begin{tabular}{|c|c|c|}
\hline $\begin{array}{c}\text { Original } \\
\text { Surface }\end{array}$ & $\begin{array}{c}\text { Traditional } \\
\text { /ms }\end{array}$ & $\begin{array}{c}\text { Improved } \\
\text { /ms }\end{array}$ \\
\hline horizontal & 258.41 & 108.29 \\
\hline vertical & 231.54 & 103.84 \\
\hline coronal & 230.26 & 103.58 \\
\hline
\end{tabular}

Under the same circumstances, the time needed by the multi-planar reconstruction based on traditional algorithm is $240.07 \mathrm{~ms}$, while the time needed by the rapid reconstruction based on the transformation of coordinates' increment. Hence, the second method of MPR display is obviously faster than the traditional one whose time is twice as much as the second method. The advantage of the second method is quite obvious, which can refresh the image more efficiently and promote the fluency and practicality of the system.

\section{CONCLUSIONS}

A portable DICOM images viewer based on iOS is developed successfully, which implements the analyses, display, transferring the window, zoom, translation and multi-planar reconstruction of DICOM images. As the portable devices possess several limitations relatively to PCs, such as low frequency, low memory and poor computing, etc, and the MPR method based on the traditional coordinate transform brings a large number of calculations, costing much time, so the improved algorithm based on the coordinates' increment is utilized here. Thus, under the premise of not changing the effects of reconstruction, the speed of the multi-planar reconstruction is greatly raised, and the fluency and operability of the system are improved, which is significant to promote the use of portable devices in the medical field and the development of health and medical undertakings.

\section{ACKNOWLEDGMENT}

The authors are grateful for grants from the National Science Fund of China(NSFC; Grant No. 61372014 , and 61201053).

\section{REFERENCES}

[1] Wang Zhi-kang,LOUHai-fang,SUNJian-fang. Application of DICOM Electronic Film in Hospital Image InformationSystem[J].China Medical Devices,2012,27(2):41-43

[2] YANG Bo-fei, LV Wei, CHU Jing-hui, YANG De-long. The Design of Android Platform-Based Medical Image Display Software[J]. Chinese Journal of Medical Physics, 2012,29(09):3375-3379.

[3] Bin Zhang, Zhi-wen Wang. Several Technical Problems of Constructing PACS[J]. Scientific Management, 2010, 25(1):7475

[4] MI Yong-wei,LI Yi-yong,CHEN Pei-xin. Design and Realization of DICOM-based Medical Image Processing Software[J]. Chinese Medical Equipment Journal,2010,31(11):69-71.

[5] National Electrical Manufacturers Association, Digital Imaging and Communication in Medicine(DICOM)[Z]. Virginia USA National Electrical Manufacture Association, 2003.

[6] OtaY, Masai A, Sato T. An Experimental Trial Of Application Multiplanar Reconstruction Method For Magnetic Resonance Imaging [C].Stockhom, Sweden:Magnetics Conference13-16 April 1993

[7] SumiakiMatsumoto,YoshiharuOhno, et al. Potential contribution of multiplanar reconstruction (MPR) to computer-aided detection of lung nodules on MDCT[J].European Journal of Radiology,2010,9(12):2083-2099

[8] NardoGimenez,JoosHeintz,Guillermo Matera et al.Lower complexity bounds for interpolation algorithms[J].Journal of complexity,2011,27(2):151-187.

[9] Shen Bin, Qi Danjin, Fan Liuqun, Zhu zhihao.Singular problem and improved plane interpolation algorithm[C]//2011 International Conference on Advanced Engineering Materials and Technology (AEMT),Sanya: Advanced Materials Research,2011: 2974-2978

[10] Philippe Thévenaz, Michael Unser.Optimization of mutual information for multiresolution image registration [J].IEEE Transactions on Image Processing,2000,9(12):2083 2099.DOI: $10.1109 / 83.887976$.

[11] GAO Zhi-yong,XUKai,LIU. Fast Transformation in Medial Image Registration[J]. Chinese Journal of Medical Instrumentation,2003,27(2):92-94 